\title{
An Experimental Comparison of Flat-Panel Detector Performance for Direct and Indirect Systems (Initial Experiences and Physical Evaluation)
}

\author{
Tsutomu Gomi, ${ }^{1}$ Kichirou Koshida, ${ }^{2}$ Tosiaki Miyati, ${ }^{2}$ Jun Miyagawa, ${ }^{1}$ and Hiroshi Hirano ${ }^{1}$
}

\begin{abstract}
The purpose of this work was to compare direct and indirect detectors in terms of their system linearity, presampled modulation transfer function (MTF), Wiener spectrum (WS), noise equivalent quanta (NEQ), and power spectrum. Measurements were made on two flat-panel detectors, GE Revolution XR/d (indirect) and Shimadzu Safire (direct) radiographic techniques. The system linearity of the systems was measured using a time-scale method. The MTF of the systems was measured using an edge method. The WS of the systems was determined for a variable range of exposure levels by two-dimensional Fourier analysis. The NEQ was assessed from the measured MTF, WS, and estimated ideal signal-to-noise ratios. Power spectrum analyzed the chest phantom within artificial lesions. System linearity was excellent for the direct systems. For the direct system, the MTF was found to be significantly higher than that for the indirect systems. For the direct system, the WS was relatively uniform across all frequencies. In comparison, the indirect system exhibited a drop in the WS at high frequencies. At lower frequencies, the NEO for the indirect system was noticeably higher than for the direct system. Power spectrum for the direct system was relatively flat and similar to that for white noise. The indirect system exhibited significant reduction at high spatial frequencies. In general, the direct systems exhibit improved image quality over indirect systems at comparable exposure dose.
\end{abstract}

KEY WORDS: Flat-panel detector, image quality, modulation transfer function (MTF), Wiener spectrum (WS), noise equivalent quanta (NEQ)

\section{INTRODUCTION}

$\mathrm{D}$ igital radiography has gained popularity in many areas of clinical practice. Computed radiography (CR) is perhaps the most abundant and common technology today with over 10,000 systems in use worldwide. In the last few years, other digital technologies, most notably the solid state-based, flat-panel detector technology, have also gained popularity. Flat-panel systems currently have a higher initial acquisition cost relative to CR. However, they offer potential for better image quality, lower radiation dose, and higher throughput. As the technology becomes more widely available and technological issues are resolved, it is expected that the cost of these systems will go down and clinical utilization will further increase.

There are currently two main types of flat-panel detectors, direct and indirect. The main difference between the two types is the conversion process. For the direct detector, a photoconductive layer, such as amorphous selenium $(\alpha-\mathrm{Se})$, converts the $x$-ray energy to electronic charges that are directed to the collecting pixel capacitors by an electric field. ${ }^{1-3}$ For the indirect detector, a scintillation phosphor layer converts the energy of $\mathrm{x}$-ray photons to visible light photons that are subsequently detected by the pixel photodiodes and stored in the form of electronic charges in the capacitors associated with each pixel. ${ }^{4-6}$ The phosphor layer may be made from granular

\footnotetext{
${ }^{1}$ From the Department of Radiology, Shinshu University Hospital, 3-1-1 Matsumoto, Nagano 390-8621, Japan.

${ }^{2}$ From the Graduate School of Medicine, Kanazawa University, 5-11-80 Kanazawa, Ishikawa 920-0942, Japan.

Correspondence to: Tsutomu Gomi, Department of Radiology, Shinshu University Hospital, 3-1-1 Matsumoto, Nagano 390-8621, Japan; tel: +81-263-354600; fax: +81-263-373375; e-mail: gomi@hsp.md.shinshu-u.ac.jp

Copyright (C) 2006 by SCAR (Society for Computer Applications in Radiology)

Online publication 14 June 2006

doi: 10.1007/s10278-006-0634-4
} 
Table 1. The imaging systems and their characteristics

\begin{tabular}{|c|c|c|c|c|c|c|}
\hline Manufacturer & $\begin{array}{l}\text { Detector } \\
\text { Type }\end{array}$ & $\begin{array}{l}\text { Detector } \\
\text { Material }\end{array}$ & $\begin{array}{c}\text { Nominal } \\
\text { Thickness }(\mathrm{mm})\end{array}$ & $\begin{array}{l}\text { Pixel Pitch } \\
\quad(\mathrm{mm})\end{array}$ & $\begin{array}{l}\text { Array } \\
\text { Size }\end{array}$ & $\begin{array}{l}\text { Imaging Area } \\
\left(\mathrm{cm}^{2}\right)\end{array}$ \\
\hline Shimadzu Co. (Sare) & Direct & $\alpha-\mathrm{Se}$ & 1.0 & 0.15 & $2,880 \times 2,880$ & $43.2 \times 43.2$ \\
\hline General Electric Co. (Revolution XR/d) & Indirect & CsI (TI) & Not disclosed & 0.2 & $2,048 \times 2,048$ & $41 \times 41$ \\
\hline
\end{tabular}

phosphor material, such as $\mathrm{Gd}_{2} \mathrm{O}_{2} \mathrm{~S}$, or phosphor materials with an oriented structure, such as cesium iodide (CsI).

The relative performance of direct and indirect digital radiographic systems influences their clinical effectiveness. The introduction of flat-panel digital radiology systems has been largely motivated by their greater dynamic range and by separation of the image acquisition and imageprocessing task, which permits good local contrast to be obtained even for regions with very different densities. Therefore, there is a need to assess and compare the performance of these systems. The performance of some direct and indirect detectors has been previously studied, focusing on the evaluation of single systems. ${ }^{7-10}$ However, there are significant methodological differences between these studies that make it difficult to directly compare their results. The purpose of this work was to assess the performance of two commercial, direct and indirect, flat-panel digital radiographic systems. The characteristics considered were the modulation transfer function (MTF), Wiener spectrum (WS), and power spectrum.

\section{MATERIALS AND METHODS}

\section{Imaging Systems}

The physical characteristics of the two systems tested are shown in Table 1. All systems were of commercial grade and had full-sized recording fields suitable for standard adult radiographic applications. The Revolution XR/d (General Electric Co.) and Safire (Shimadzu Co.) systems were installed in the Radiology Department at Shinshu University. Before initializing the measurements, all two systems were calibrated (detector offset and gain correction) without grid according to the guidelines from the manufacturer. For all data acquisitions, the images were transferred as raw, unprocessed data to our research computers via CD-R media.

\section{Measurement of Fundamental Physical Imaging Properties}

We measured and evaluated system response, presampling MTF, and digital WS for the direct and indirect systems based on IEC62220-1. ${ }^{11}$ We obtained linear original images, which were preprocessed with offset and gain corrections, as shown in Figure 1, to neglect the postprocessing effects. All measurements used x-ray sources with high-frequency generations, a small focal spot, and a source-to-detector distance of $200 \mathrm{~cm}$. There was a minimum of 1-min delay between acquisitions to minimize the contribution of any potential lag signal in the acquired data.

Response of the systems was verified within the tested exposure range. At each beam quality, multiple uniform images were acquired using different exposures (time-scale method). For each image acquisition, a calibrated ion chamber was positioned (upper position at $50 \mathrm{~cm}$ ) before the receptor. The chamber was positioned so that it projected over the center of the detector. We use the same beam quality in the measurements for system response, MTF, and WS (half-value layer $7.1 \mathrm{~mm} \mathrm{Al}$, additional filtration $21 \mathrm{~mm}$, and approximate x-ray tube voltage $70 \mathrm{kV}$ ).

The resolution properties of the direct and indirect systems were evaluated by measuring MTF. To obtain the inherent resolution property in the direct and indirect systems, presampling MTF was obtained by a two-dimensional Fourier transformation of the edge images. ${ }^{12}$ In the radiography of edge images, we used a conventional x-ray unit (AUD150G,

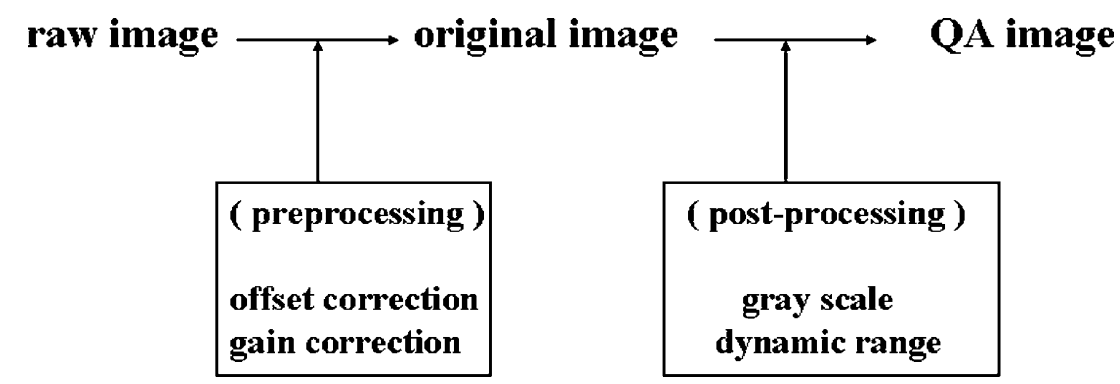

Fig 1. Linear original imaging preprocesses with offset and gain correction except for the postprocessing effects. 
Shimadzu, Kyoto, Japan). A slightly angular tungsten alloy edge (usually $<3^{\circ}$ ) of $100 \times 100 \times 1 \times \mathrm{mm}$ was used to obtain the edge image at different alignments between the center of the edge and the sampling coordinate. The exact angle of the edge with respect to the pixel lattice was determined from a least-squares fit to the edge transition position. To eliminate geometric anomalies, the edge was placed directly at the center of the detector surface. A system response characteristic curve, which relates the output pixel value to the input relative $\mathrm{x}$-ray intensity, was used as a means of linearization. The MTF was obtained by a fast Fourier transformation of the line spread function, the derivative of the edge spread function.

The WS was assessed according to the IEC62220-1 standard. The physical properties of radiographic noise for direct and indirect systems were quantified by means of the WS. The WS was obtained using the fast Fourier transformation of a two-dimensional noise pattern of a uniform-exposure image obtained at $70 \mathrm{kV}$. Incident exposure was accurately measured by using an ionization chamber mounted in a fixed position between the focal spot and the detector. The measured $\mathrm{WS}$, in terms of pixel value, were converted to those in terms of relative $\mathrm{x}$-ray intensity by using the system response characteristic curve.

The noise equivalent quanta (NEQ) ${ }^{13,14}$ was calculated from the presampling MTF and the normalized WS according to its usual definition. It characterizes the image quality by describ- ing the virtual fluence of photons that a quantum-limited detector would have required to produce the same image information.

$$
\begin{aligned}
& N E Q(u)=\frac{[\operatorname{MTF}(u)]^{2}}{N W S(u)} \\
& N W S(u)=\frac{W S(u)}{\left(\gamma \log _{10} e\right)^{2}}
\end{aligned}
$$

$\operatorname{MTF}(u)$ is measured by presampled MTF. The normalized WS [NWS $(u)$ ] is the WS divided by the gain of the system, which is determined by the gamma value $\gamma$ for system linearity.

A schematic diagram of a power spectrum analysis is shown in Figure 2. To simulate the clinical conditions of a human body, a chest phantom was used (Kyoto Kagaku Co., Tokyo, Japan). Artificial lesions were lower field within the predefined regions. The radiation dose was set at almost similar values for the direct and indirect systems (direct system $0.17 \mathrm{mGy}$, indirect systems $0.14 \mathrm{mGy}$ ).

\section{RESULTS}

All systems demonstrated excellent linearity (Figs. 3 and 4). The pixel values in the Safire
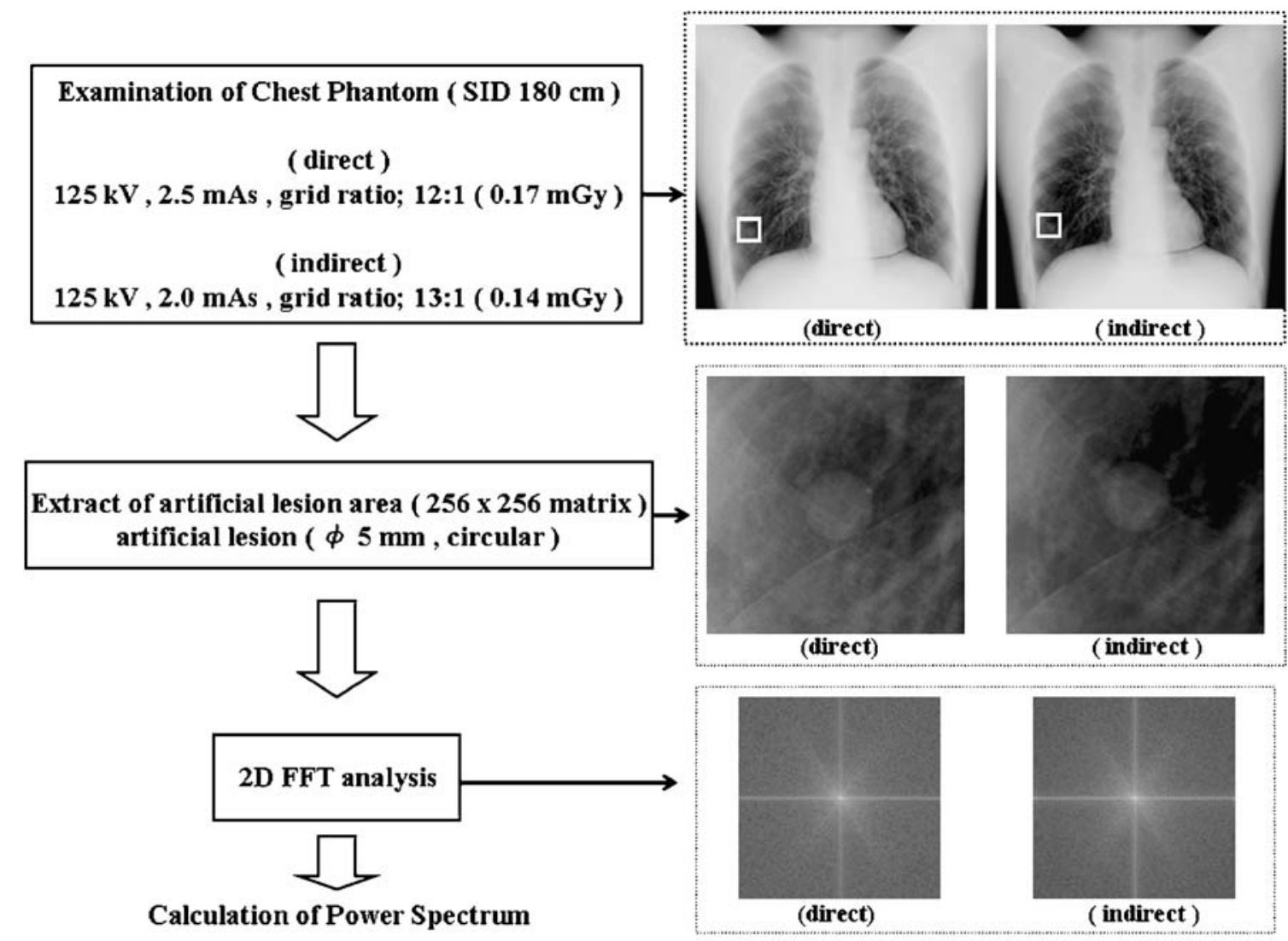

Fig 2. Flow diagram of the power spectrum analysis. 


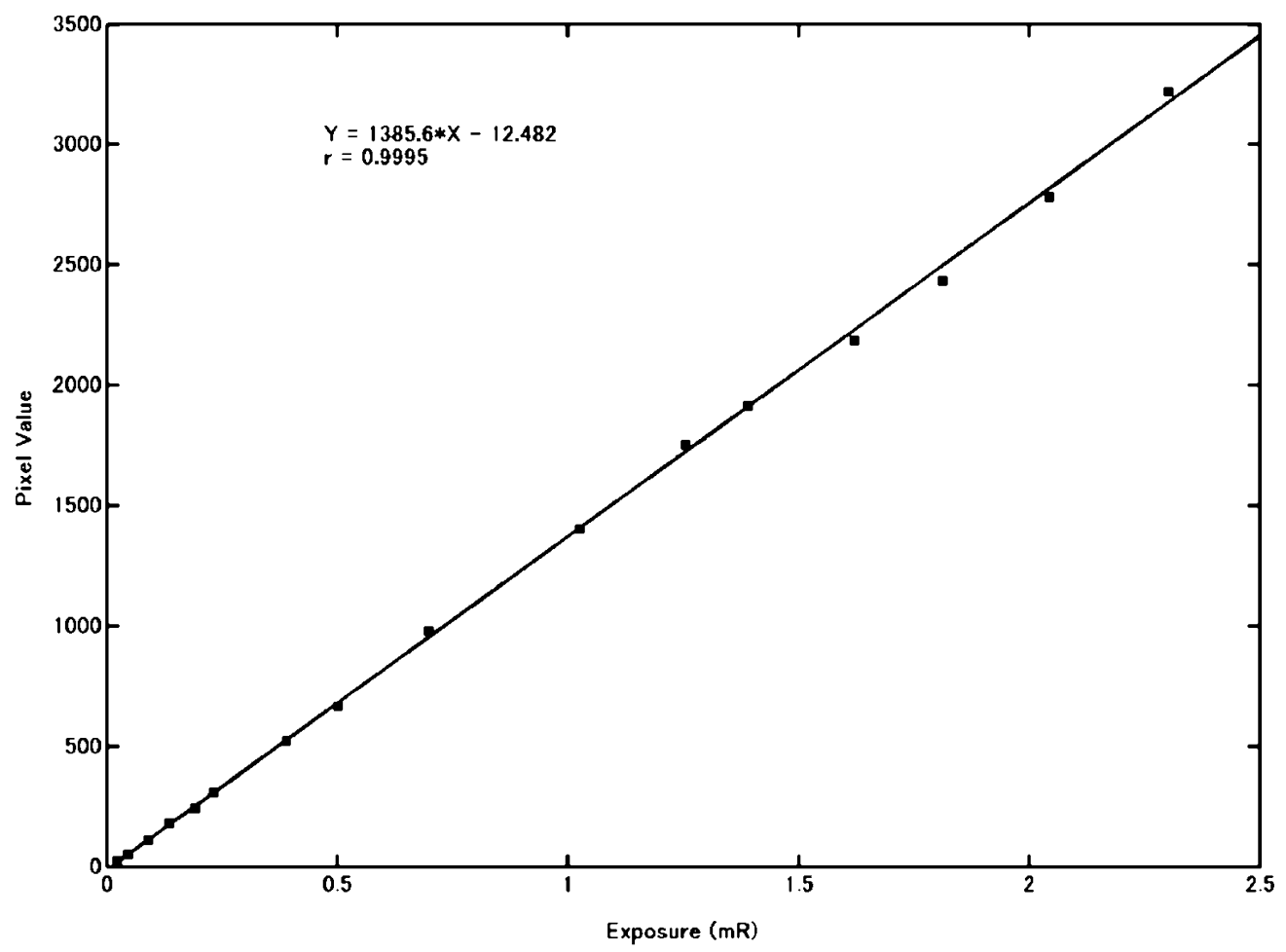

Fig 3. The relationship between the pixel value and exposure for the Sare (direct) system using the time-scale technique.

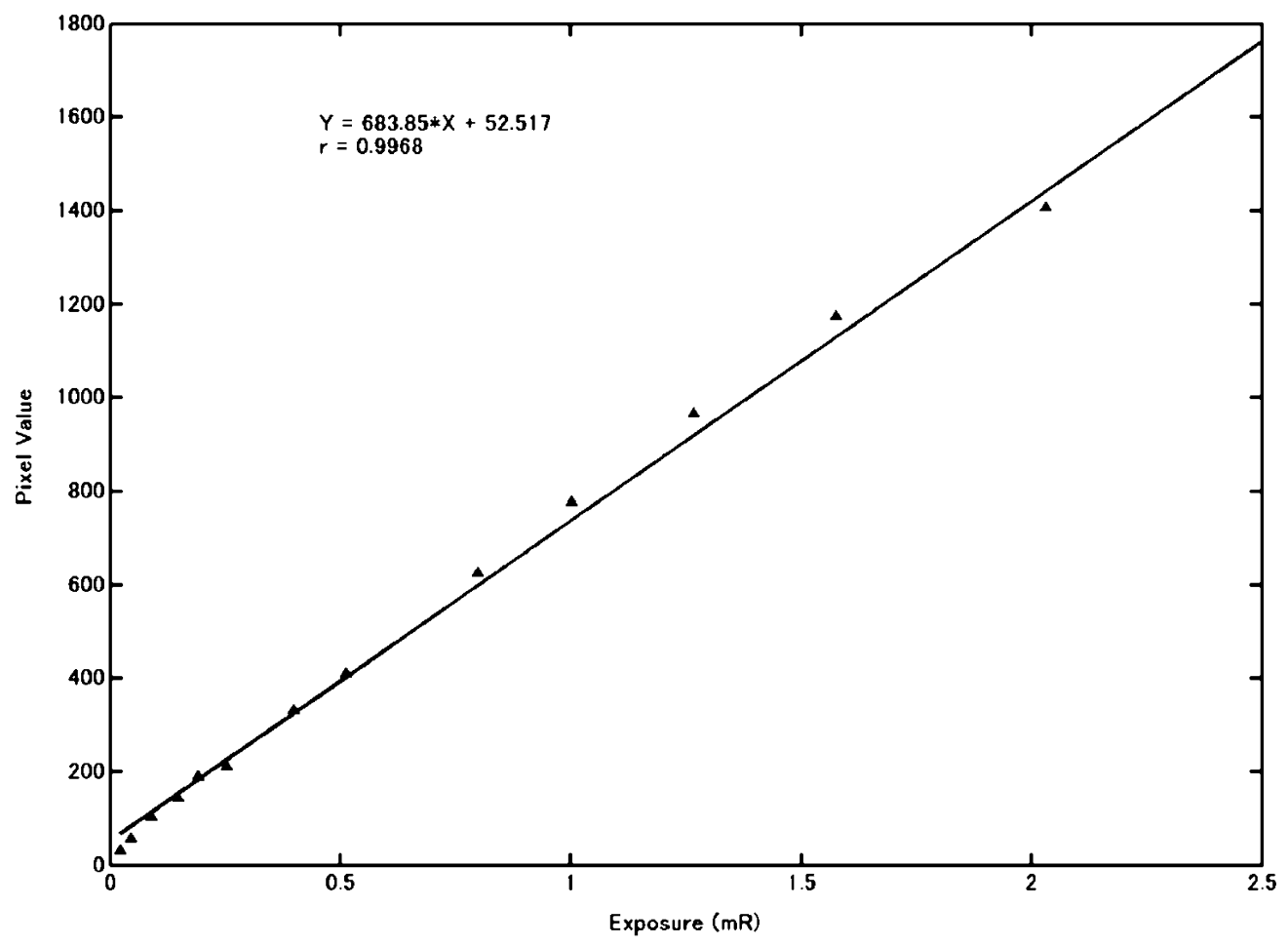

Fig 4. The relationship between the pixel value and exposure for the XR/d (indirect) system using the time-scale technique. 
and XR/d systems exhibited a linear relationship with exposure and were proportional to the logarithm of exposure. The relationships were used to linearize the image data with respect to exposure with zero offset, an important requirement of linear system analysis.

The MTF determined for the direct detector system was notably higher than the MTF for the indirect detector system (2\% MTF value; direct 5.6 cycles $/ \mathrm{mm}$, indirect 2.6 cycles $/ \mathrm{mm}$; Fig. 5). Figure 6 shows the comparison of WS for the two systems. The WS of the Safire direct detector was relatively flat and similar to that for white noise. The XR/d system exhibited significant reduction at high spatial frequencies. Figure 7 shows the comparison of the NEQ for the two systems. Compared with the Safire, the NEQ of the XR/d system was notably higher at lower frequencies but dropped more rapidly at higher spatial frequencies. For the Safire system, the NEQ at mid to high spatial frequencies were higher. Figure 8 shows that the power spectrum for the
Safire system was relatively flat and similar to that for white noise. The XR/d system exhibited significant reduction at high spatial frequencies. As for the noise (white noise) to be added to a signal component from these tendencies, the Safire system can do more than the XR/d system.

In addition, we present clinical cases from the same patient (Figs. 9 and 10).

\section{DISCUSSION}

Digital radiography using solid-state detectors is emerging as a viable technology for acquiring digital x-ray images. Many manufacturers now offer medical imaging systems based on this technology. However, there are important differences in the particular implementations of the technology, most notably in the use of photoconductor-based (ie., direct) or photosphere-based approaches for x-ray detection. Ultimately, the utility of these approaches should be examined by

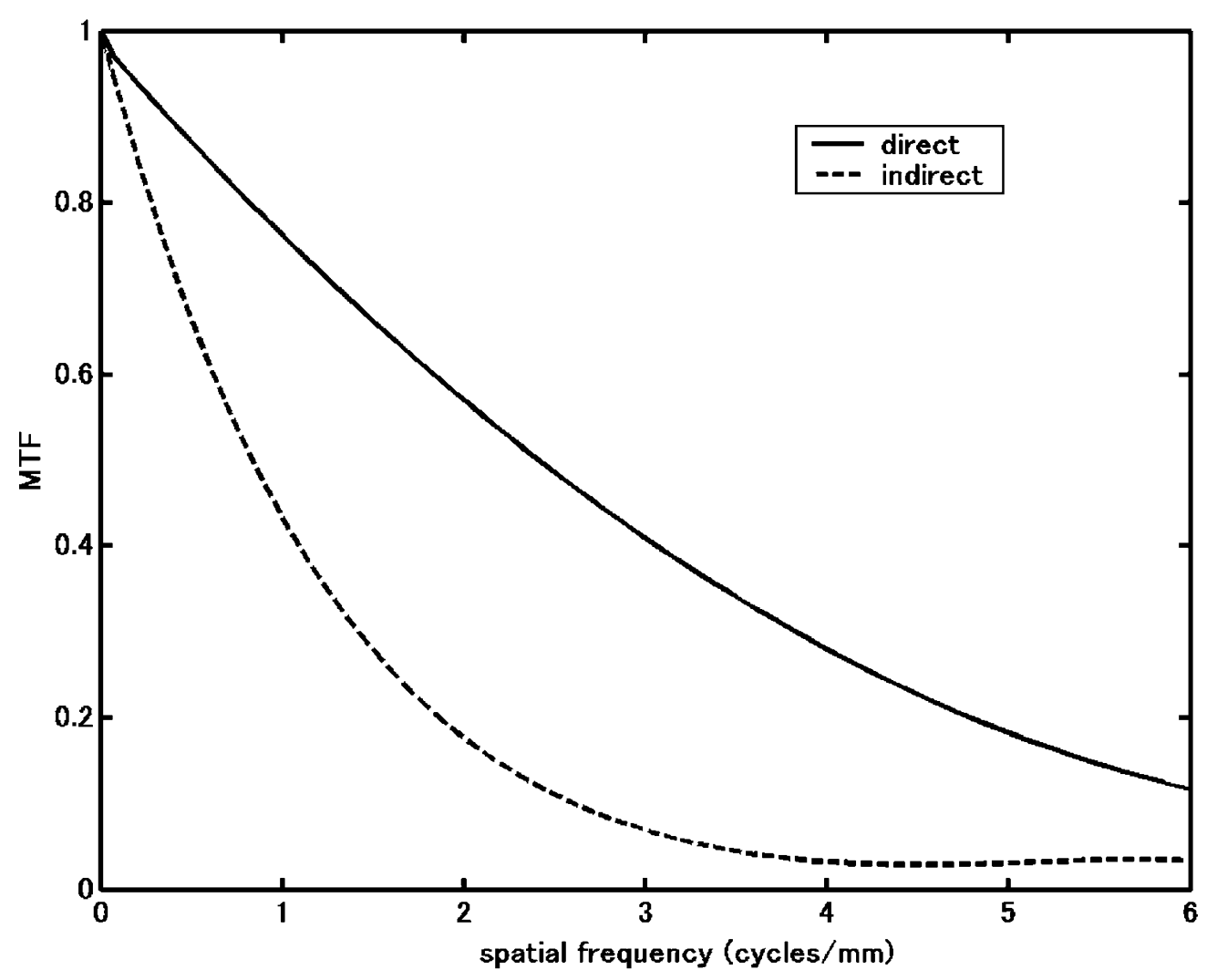

Fig 5. Comparison of presampling MTFs for the Sare (direct) and XR/d (indirect) systems. 


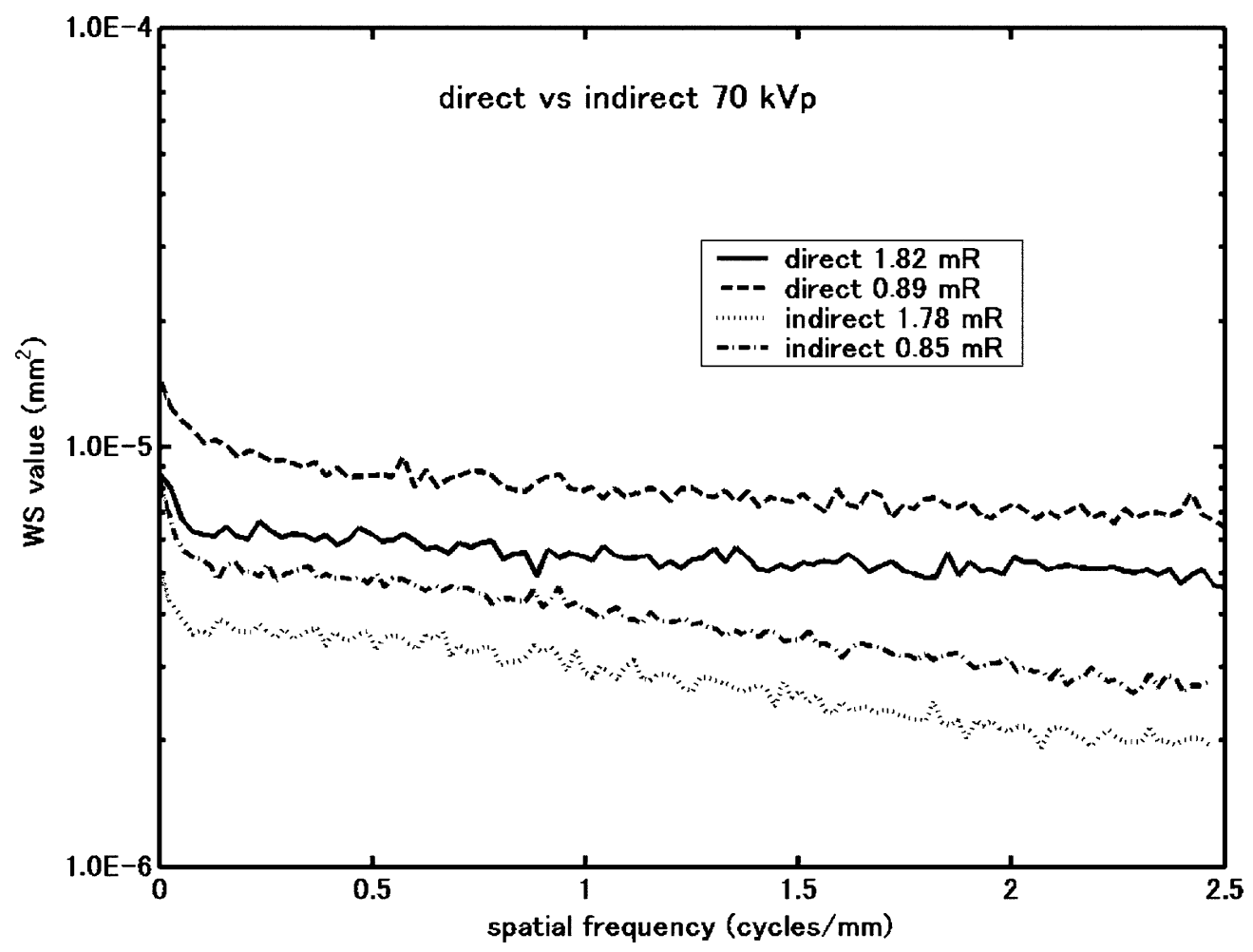

Fig 6. Comparison of WS in the vertical directions for the Sare (direct) and XR/d (indirect) systems.

clinical trials. In the absence of clinical trial results, the key physical attributes of these systems can be evaluated experimentally and used to predict the clinical efficacy of various implementations of the technology. In this work, we have compared the resolution (MTF), noise (WS), and signal-tonoise ratio (NEQ) characteristics of direct and indirect flat-panel detector systems.

The MTF determined for the direct detector system was notably higher than the MTF for the indirect detector system. Table 2 compares the results. For the Safire detector (direct detector), the charge is collected with little spread and thus good resolution is expected relative to the indirect detection systems where light scattering causes blur. The resolution response of the $\mathrm{XR} / \mathrm{d}$ system was seen to be similar to that of the CR system.

At higher spatial frequencies, the resolution properties of the direct system may contribute to the higher NEQ, such as the MTF in the direct system. However, the situation is not straightforward for the WS in the higher spatial frequencies. Lubberts ${ }^{16}$ described that $\mathrm{x}$-rays deposited at various depths within the scintillator layer could show different amounts of spreading before they reached the surface of the indirect detector. This concept states that $\mathrm{x}$-rays with identical energy would give different point spread functions (PSF) depending on the deposition depths. This phenomenon suggests that a detection process at the scintillator could cause the noise because $\mathrm{x}$-rays with identical energy, which should be detected identically, would be detected differently. This would affect the propagation of signal and noise differently. The former is characterized as MTF and computed using an average of PSF from each layer, whereas the latter is characterized as a noise transfer function and computed using a root square sum of PSF from each layer. Lubberts showed that this mechanism, now known as the Lubberts effects or the optical Swank factor, decreases the WS as spatial frequencies increase. ${ }^{17}$

The actual clinical performance of the various systems depends on many factors other than NEQ, including the operating exposure ranges for the acquisition of clinical images, detector sensitivity to scattered radiation, the use of antiscatter grids, and image processing. Nevertheless, assuming 


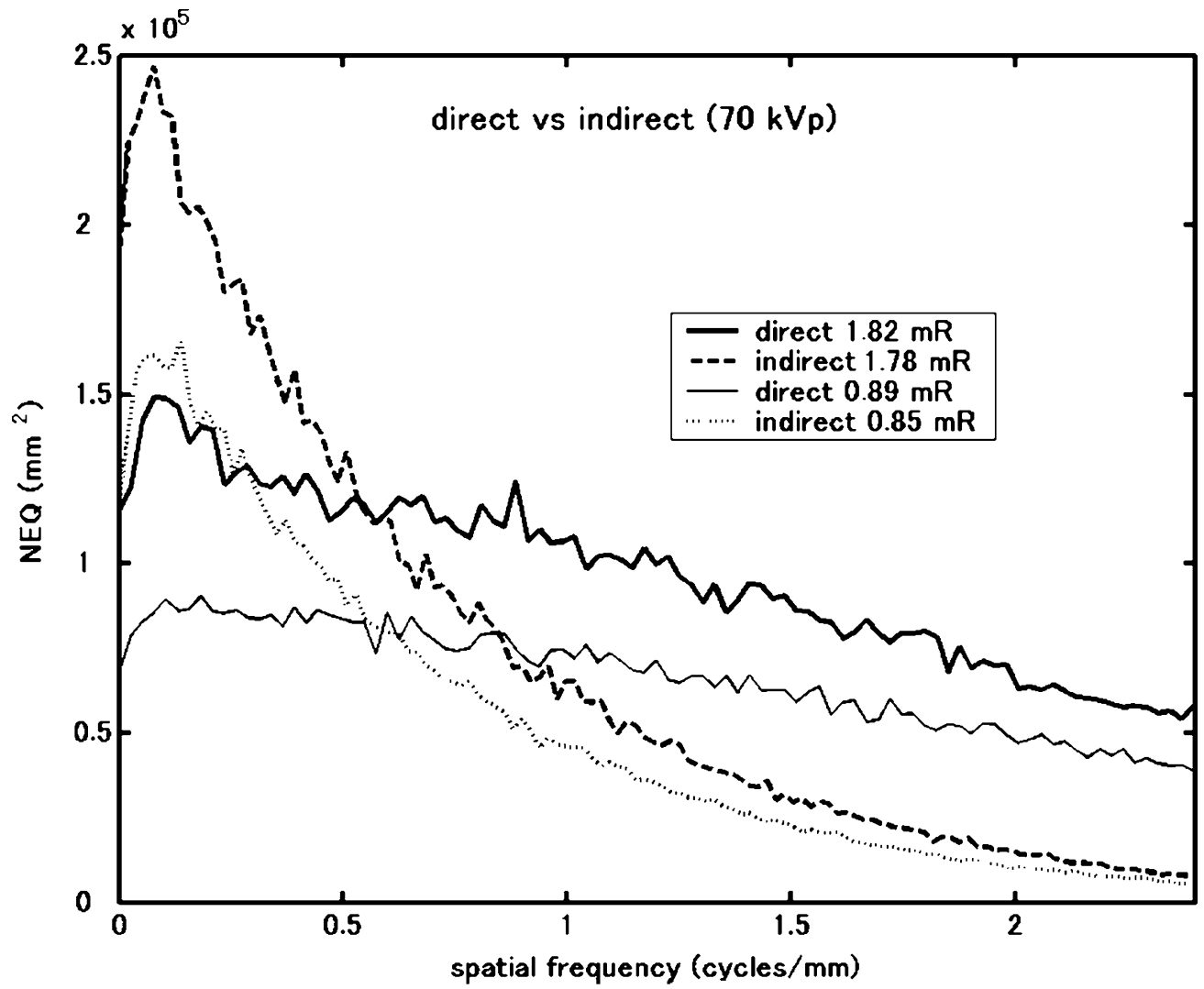

Fig 7. Comparison of NEQ for the Sare (direct) and XR/d (indirect) systems.

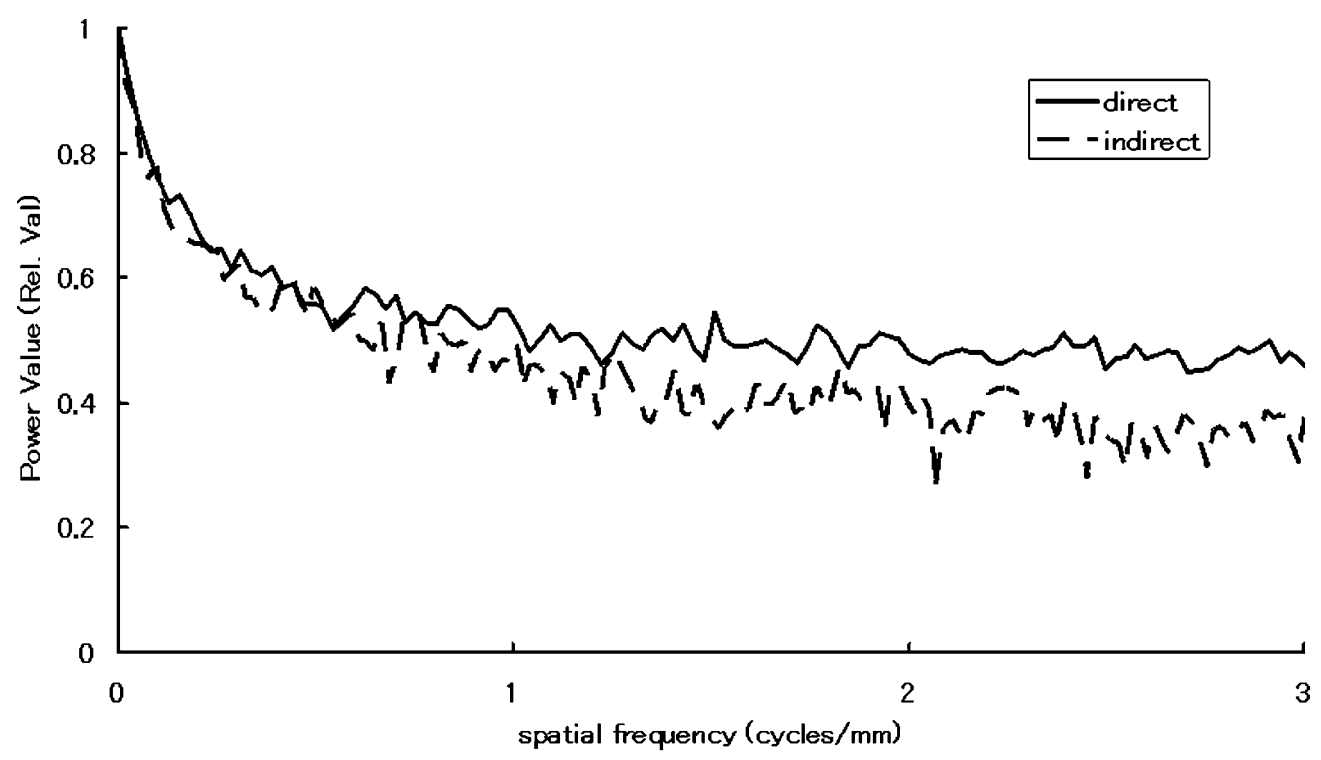

Fig 8. Comparison of power spectrum for the Sare (direct) and XR/d (indirect) systems. 


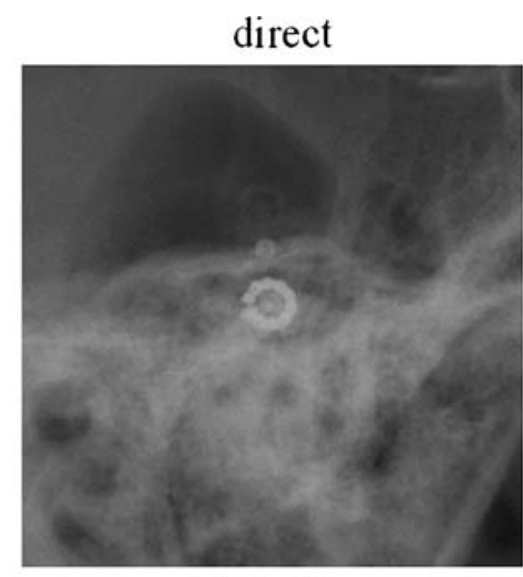

$1.93 \mathrm{mGy}$

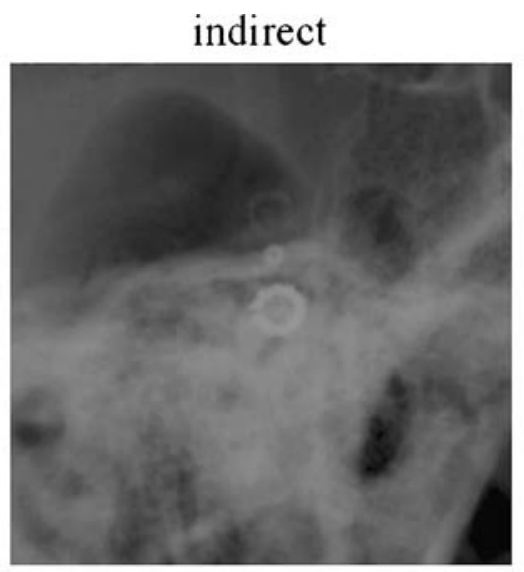

$1.55 \mathrm{mGy}$

$(70 \mathrm{kV}$. 20mAs. SID $150 \mathrm{~cm}$. grid ratio $5: 1)(70 \mathrm{kV}, 20 \mathrm{mAs}$. SID $150 \mathrm{~cm}$. grid ratio $5: 1)$

Fig 9. Example of clinical examination, case 1. Comparison of head radiography for the Sare (direct) and XR/d (indirect) systems.

similar patient exposures, some general implications can be drawn from the comparison of the results on the direct and indirect systems that were tested in this study. The high MTF and superior NEQ of the direct system above $\sim 2.0 \mathrm{~mm}^{-1}$ suggest that this system may be particularly effective in radiographic applications where fine anatomic structures need to be imaged with high detail and contrast. Utilization of this system for

\section{direct}

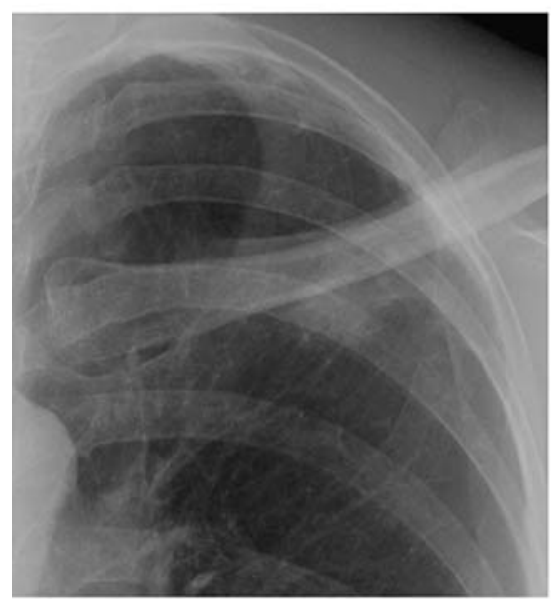

$0.17 \mathrm{mGy}$

$(125 \mathrm{kV}, 2.50 \mathrm{mAs}, \mathrm{SID} 180 \mathrm{~cm}$. grid ratio 12:1) imaging trabecular bone structures in skeletal extremities, for example, would thus be indicated. On the other hand, the extremely high NEQ of the direct system at frequencies below $2.0 \mathrm{~mm}^{-1}$ makes it attractive in radiographic applications where the visibility of low-contrast anatomic structures is limited by noise. Utilization of these systems for imaging of lung nodules in thoracic imaging, for example, would thus be indicated.

\section{indirect}

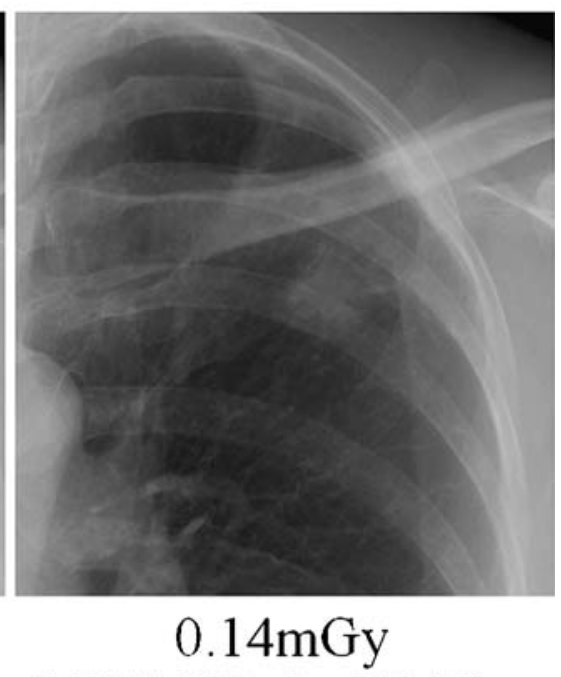

(125kV, $2.03 \mathrm{mAs}$, SID $180 \mathrm{~cm}$. grid ratio 13:1)

Fig 10. Example of clinical examination, case 2. Comparison of chest radiography for the Sare (direct) and XR/d (indirect) systems. 
Table 2. MTF Results

\begin{tabular}{cccc}
\hline MTF & Direct $\left(\mathrm{mm}^{-1}\right)$ & Indirect $\left(\mathrm{mm}^{-1}\right)$ & $\mathrm{CR}^{\mathrm{a}}\left(\mathrm{mm}^{-1}\right)$ \\
\hline 0.1 & 6.3 & 2.5 & 3.4 \\
0.2 & 4.8 & 1.8 & 2.4 \\
\hline
\end{tabular}

${ }^{\mathrm{a}}$ FUJI, FCR-9501-HQ, ST-Va, $0.1 \mathrm{~mm}$ pixel, from Samei and Flynn. ${ }^{15}$

Results are summarized by tabulating the average of the response. For comparison, the results for a typical CR system are also shown.

However, the relative significance of high and low frequencies for particular clinical tasks and the clinical implications of signal and noise aliasing in the direct systems await further investigations.

\section{REFERENCES}

1. Yaffee MJ, Rowlands JA: X-ray detectors for digital radiography. Phys Med Biol 42:1-39, 1997

2. Lee DL, Cheung LK, Rodricks BG, et al: Improved imaging performance of a $14 \times 17$-inch digital radiography system using Se/TFT detector. Proc SPIE 3336:14-23, 1998

3. Zhao W, Blevis I, Germann S, et al: Digital radiography using active matrix readout of amorphous selenium: construction and evaluation of a prototype real-time detector. Med Phys 24:1834-1843, 1997

4. Siewerdesn JH, Antonuk LE, EI-Mohri Y, et al: Empirical and theoretical investigation of the noise performance of indirect detection, active matrix flat-panel imagers (AMFPIs) for diagnostic radiography. Med Phys 24:71-89, 1997

5. Chaussat C, Chabbal J, Ducourant T, et al: New CsI/a-Si $17^{\prime \prime} \times 17^{\prime \prime} \mathrm{x}$-ray flat-panel detector provides superior directivity and immediate direct digital output for general radiography systems. Proc SPIE 3336:45-56, 1998
6. Colbeth RE, Cooper VN, Gilblom DL, et al: Characterization of a third-generation multimode sensor panel. Proc SPIE 3659:491-500, 1999

7. Granfors PR, Aufrichtig R: Performance of a $41 \mathrm{~cm} \times 41$ $\mathrm{cm}$ amorphous silicon flat panel $\mathrm{x}$-ray detector for radiographic imaging applications. Med Phys 27:1324-1331, 2000

8. Floyd CE, Warp Jr, RJ, Dobbins JT, et al: Imaging characteristics of an amorphous silicon flat-panel detector for digital chest radiography. Radiology 218:683-688, 2001

9. Launders JH, Kengyelics SM, Cowen AR: A comprehensive physical image quality evaluation of a selenium based digital x-ray imaging system for thorax radiography. Med Phys 25:986-997, 1998

10. Gingold EL, Lee DL, Jeromin LS, et al: Development of a novel-resolution direct conversion $\mathrm{x}$-ray detector. Proc SPIE 3977:185-193, 2000

11. IEC62220-1: Medical electrorical equipment-characteristics of digital X-ray imaging devices-Part 1: Determination of the detective quantum efficiency, International Electrotechnical Commission, Switzerland, 2003

12. Samei E, Flynn MJ, Reimann DA: A method for measuring the presampled MTF of digital radiographic systems using an edge test device. Med Phys 25:102-113, 1998

13. Fujita $H$, Ueda $K$, Morishita $J$, et al: Basic imaging properties of a computed radiographic system with photostimulable phosphors. Med Phys 16:52-59, 1989

14. International Commission on Radiation Units and Measurements: Medical imaging - the assessment of image quality, ICRU Report No. 54, International Commission on Radiation Units and Measurements, Bethesda, MD, 1996

15. Samei E, Flynn MJ: An experimental comparison of detector performance for computed radiography systems. Med Phys 29:447-459, 2002

16. Lubberts G: Random noise produced by $\mathrm{x}$-ray fluorescent screens. J Opt Soc Am 58:1475-1483, 1968

17. Beutel J, Kundel HL: Handbook of Medical Imaging, vol. 1. In: Van Metter RL ed Physics and Psychophysics. SPIE, Bellingham, WA, 2000 